\title{
EL MANICOMIO PROVINCIAL DE MÁLAGA EN EL PRIMER TERCIO DEL SIGLO XX: LA UTOPÍA QUE (NO) PUDO SER
}

\author{
Celia Garcia-Diaz \\ Área de Historia de la Ciencia / Universidad de Málaga \\ celiagarciad@uma.es \\ ORCID iD: https://orcid.org/0000-0003-2132-9502
}

Recibido: 25 agosto 2017; Aprobado: 16 abril 2018.

Cómo citar este artículo/Citation: Garcia-Diaz, Celia (2018), “El Manicomio Provincial de Málaga en el primer tercio del siglo XX: la utopía que (no) pudo ser", Asclepio, 70 (2): p238. https://doi.org/10.3989/asclepio.2018.22

RESUMEN: Durante el primer tercio del siglo XX en España acontecieron cambios importantes en la asistencia a la locura. Varios factores influyeron en el desarrollo de las modificaciones en torno al discurso y práctica de una nueva disciplina psiquiátrica: una generación de médicos interesados en la locura y relacionados con la Junta de Ampliación de Estudios, organizaciones científicas como los Archivos de Neurobiología y el clima político progresista de la Segunda República, entre otros. El objetivo de este trabajo es visibilizar estrategias de cambio en el tratamiento de la locura en el psiquiátrico provincial de Málaga. Para ello, señalaré, por un lado, las diferentes reformas que el edificio necesitó y la relación de éstas con las prácticas asistenciales; y por otro, los intentos de reforma que llevaron a cabo Miguel Prados Such y Pedro Ortiz Ramos como profesionales de la neuropsiquiatría. Analizaré, finalmente, las relaciones entre el personal subalterno, los psiquiatras y la institución, mostrando las dinámicas de asimilación y/o rechazo de medidas concretas que pretendían mejorar las condiciones de los pacientes ingresados.

PALABRAS CLAVE: Historia de la psiquiatría; Segunda República Española; manicomio; Málaga; Miguel Prados Such; Pedro Ortiz Ramos.

\section{THE MALAGA PROVINCIAL MENTAL ASYLUM (SPAIN) IN THE FIRST THIRD OF THE 20TH CENTURY: THE UTOPIA THAT WAS (NOT) TO BE}

ABSTRACT: During the first third of the 20th Century in Spain, many reforms happened in the attendance to the madness. Several factors had an influence in the introduction of fundamental changes in the discourse and practice of a new psychiatric discipline: the interest in insanity of a generation of doctors with links to the Junta de Ampliación de Estudios (Board for Advanced Studies), scientific organisations like the Archivos de Neurobiología (Archives of Neurobiology) and the political climate in the Second Spanish Republic, among others. The aim of this study is to shed light on these strategies for change in the treatment of insanity in the Malaga provincial psychiatric hospital. To this end, I will discuss the necessary alterations made to the building itself and their relation with health care practices, in addition to the attempts to introduce reforms by neuropsychiatric professionals like Miguel Prados Such and Pedro Ortiz Ramos. Finally, I will analyse relations among support staff, psychiatrists and the institution, to appraise the dynamics of assimilation and/or rejection of specific measures designed to improve the conditions of patients admitted to it.

KEY WORDS: History of psychiatry; Second Spanish Republic; asylum; Málaga; Miguel Prados Such; Pedro Ortiz Ramos. 


\section{INTRODUCCIÓN}

La relación entre arquitectos y médicos alienistas fue fundamental para la creación de nuevos espacios para la locura durante el siglo XIX, siendo objeto de recientes investigaciones en España (Navarro, 2015). Sin embargo, en el intento de los primeros alienistas en romper las cadenas de los enfermos, se gestó la creación de manicomios con puertas cerradas. Esquirol escribió Des maladies mentales considérées sous les rapports médical, hygiénique et médico-legal (Esquirol, 1838) ${ }^{1}$ donde recomendaba la clasificación de los enfermos mentales, según su estado, dentro de la estructura manicomial. Así, defendía una forma ideal de manicomio diseñado en 1833 por Lebas, con un edificio central para servicios generales y habitaciones de médicos y enfermeros; perpendicularmente, y a ambos lados, quedarían los pabellones destinados a los enfermos, donde los alienados serían clasificados, y cuyo número y colocación dependería del tipo de conducta y su duración (Huertas, 2008, p. 123-140). La influencia de los Falret en nuestro país fue importante, como Jose Luis Peset muestra en su trabajo "El manicomio modelo en España” (Peset, 1995, p. 43-52; Falret, 1852; Falret, 1864). Las revistas médicas españolas de mediados del XIX comenzaron a interesarse por la cuestión de la locura, publicando artículos donde se mezclaban taxonomías psiquiátricas en un intento de clasificación científica, y llegaba a establecerse el número de dementes que debían ocupar cada estancia en los establecimientos manicomiales (Álvarez-Uría, 1983, p. 116-117). Fue a partir de 1846 cuando se inició el debate en torno a la asistencia de los enajenados en un clima de interés por la construcción de instituciones donde poder controlar la furia de los locos que ponía en peligro la sociedad burguesa en plena industrialización. Para ello, se elaboraron las primeras estadísticas sobre dementes en junio de 1848, en las que Málaga ocupaba el quinto lugar en número de enfermos ${ }^{2}$, después de Barcelona, Jaén, Castellón y Zaragoza (Álvarez- Uría, 1983, p.120). En 1859 casi se duplicó el número de enajenados, y en la estadística realizada en 1879 a nivel nacional, se objetivó que el número de enajenados ingresados se había triplicado desde 1860 (González Duro, 1996, p.22). En la estadística de manicomios de los años 1879-1880 figuraba por primera vez una institución malagueña, el Hospital Nuestra Señora de los Ángeles ${ }^{3}$, adscrita a la Diputación Provincial (Rodríguez-Méndez, 1880, p.655). En Andalucía, como en el resto de España, la asistencia a los dementes estaba en manos de las órdenes religiosas hasta la mitad del siglo XIX, donde, gracias a la ley de la Beneficencia de 1842, comenzó a generarse el debate en torno a la construcción de los manicomios modelos. Varios estudios sobre las instituciones del sur del país ponen de manifiesto los procesos de creación de los manicomios de la Beneficencia en diferentes provincias ${ }^{4}$, si bien en el caso de Málaga hay un vacío historiográfico en torno a la institución, sus profesionales, y cómo se organizó la asistencia a los enfermos, en un periodo marcado por profundos cambios sociales y políticos. En este trabajo mostraré cómo fue construido el Manicomio Provincial de Málaga y cómo se organizó el espacio, para centrarme posteriormente en los profesionales que fueron directores de la institución psiquiátrica en las décadas de los 20 y los 30 (Miguel Prados Such y Pedro Ortiz Ramos), y su influencia en la elaboración de diferentes proyectos que he denominado "utopía asistencial", cambios que propiciaron reformas asistenciales, y por tanto, estructurales en el manicomio de Málaga durante la Segunda República que se vieron truncados por la Guerra Civil. Por último, señalaré cómo el interés por cambiar las prácticas dentro del manicomio, a través de la formación de enfermeros especializados, chocaron frontalmente con el antiguo personal subalterno y la tendencia a mantener el status quo por parte de la Diputación Provincial de Málaga.

\section{ESPACIO MANICOMIAL EN MÁLAGA: CONSTRUC- CIÓN Y REFORMAS DE UN HOSPITAL DE PABELLONES}

A finales del siglo XIX la asistencia sanitaria en Málaga era muy precaria y recaía fundamentalmente en órdenes religiosas, por lo que la construcción del pabellón manicomial de San Carlos, integrado en el recinto del Hospital Civil Provincial de Málaga ${ }^{5}$, aunque separado del edificio principal, supuso un cambio significativo en la atención a los dementes de la provincia. Las obras del manicomio se iniciaron en el marco de la construcción del Hospital Civil y, aunque no hay una fecha de inicio clara del pabellón manicomial en los documentos consultados, se sabe que las obras sufrieron también numerosas interrupciones por falta de fondos de la Beneficencia (Fernández, 2004, p.362-418). Por ello, las donaciones de familias burguesas que residían en Málaga fueron cruciales para el desarrollo de las obras del hospital en general y del pabellón manicomial en particular ${ }^{6}$. Previo a su construcción, la falta de infraestructura para la asistencia psiquiátrica provocó que enfermos de la provincia de Málaga tuviesen que ser ingresados en la casa Hospicio de Granada ${ }^{7}$ hasta 1864 , fecha en la que la Beneficencia inauguró el "Departamento de observación de dementes del lazareto de los Ángeles"8 (Delange, 2003, p.226), un espacio habilitado para los dementes hasta que finalizara la construcción del manicomio. El retraso en las obras del pabellón manicomial San 
Carlos continuó dificultando la asistencia psiquiátrica en la provincia, de forma que 22 enfermos ingresados en el Departamento de observación de dementes del Asilo de los Ángeles esperaban ser trasladados al Manicomio de San Baudilio de Llobregat en el año $1877^{9}$.

Debido al peligro de contagio durante la epidemia de cólera que estaba sufriendo la población en 1885, los enfermos que seguían ingresados en el Departamento de observación de dementes de los Ángeles, un total de 120, fueron trasladados urgentemente al entonces nuevo Hospital Civil (Delange, 2003, p.431). Al no encontrarse aún disponible el recinto manicomial, se ubicaron en la enfermería llamada San Antonio, en el edificio principal del hospital, donde la asistencia psiquiátrica y las medidas de seguridad eran inexistentes ${ }^{10}$ (Fernández, 2004, p. 389).

En estas fechas los médicos que se hacían cargo de la asistencia a dementes no tenían formación especializada, y se ocupaban fundamentalmente de los problemas orgánicos o enfermedades concomitantes que podían padecer los ingresados en el manicomio. Esta práctica no sólo ocurría en Málaga, sino que era habitual en otros muchos establecimientos psiquiátricos
(Campos y Huertas, 1998, p. 99). El 31 de diciembre de 1898 finalizó la construcción del Manicomio Provincial, pero los pacientes no ingresaron hasta el 20 de marzo de 1899. José Delgado Collantes y Francisco Linares Enríquez ${ }^{11}$ figuran en los libros de personal como los primeros "médicos de enfermedades nerviosas" que se encargaron de atender a los enfermos desde 1889. Miguel Prados Such, que se incorporó a la institución en 1925, fue el primer médico que se interesó por la práctica de una ciencia neuropsiquiátrica nueva en el Hospital Civil. Dos años más tarde, Pedro Ortiz Ramos, también como profesor del cuerpo médico de la Beneficencia provincial, se sumó a un ejercicio profesional especializado.

El Manicomio de San Carlos era un edificio sencillo y simétrico, con dos alas diferenciadas donde albergar dementes hombres y mujeres. Cada ala estaba dividida en diferentes espacios organizados según la nosología psiquiátrica del momento, aunque, como apunta Josep Comelles, se trataba más de una clasificación social que nosológica (Comelles, 1988, p.59). Así, existían dependencias para convalecientes, sucios, agitados, y otro espacio dividido en estancias individuales para los furiosos (Fernández, 2004, p.390) (ver figura 1).

Figura 1. ADPM. Planta del manicomio de San Carlos, (7-5-1896), p. 5:35

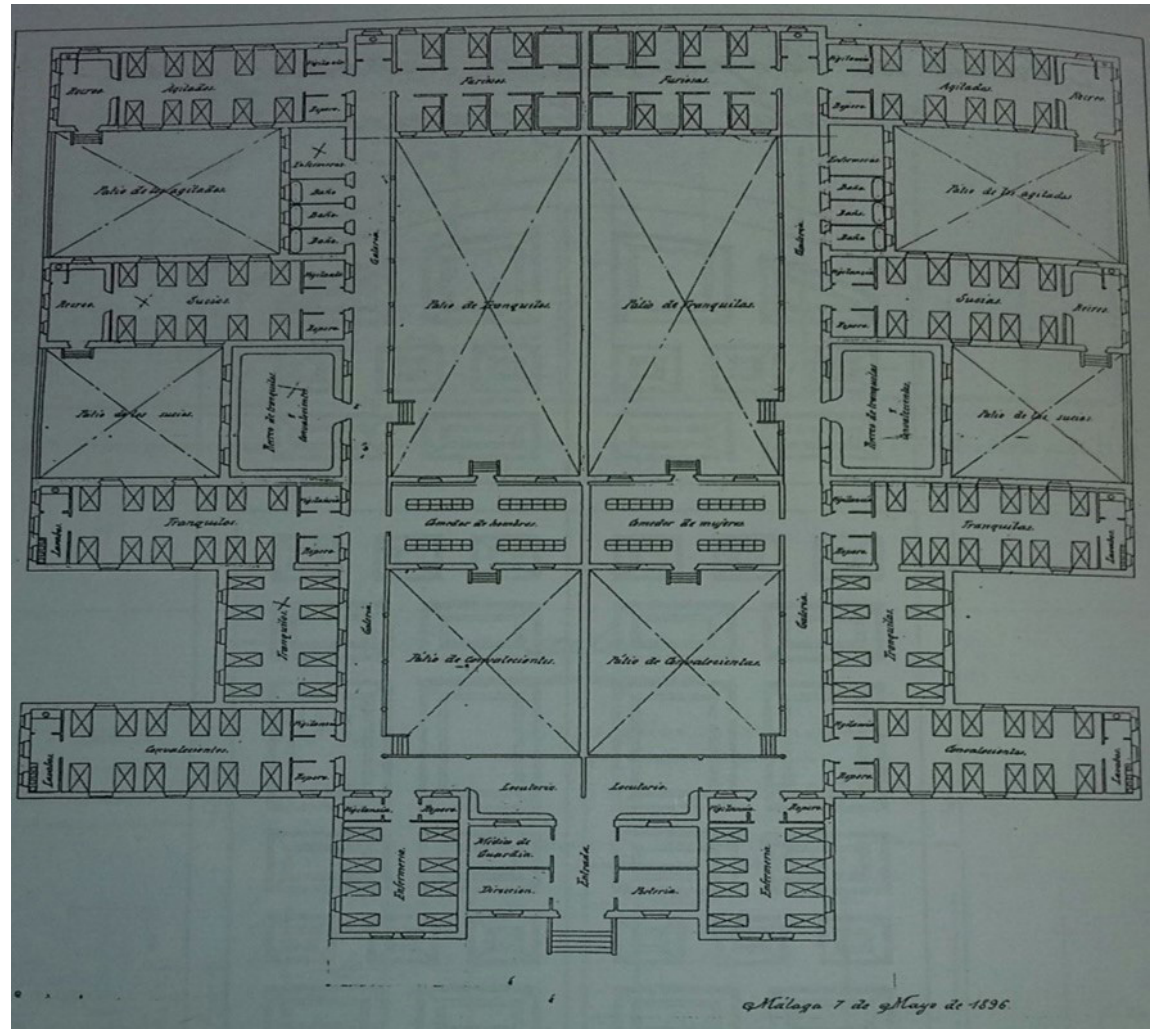


Para ser admitidos, los enfermos debían presentar el certificado de pobreza y acreditar su residencia en Málaga, en la Comisaría de entrada. El ingreso se decidía en la Comisión o Pleno Provincial, que dependía de la Diputación, exceptuando los ingresos judiciales. En el reglamento del Hospital Civil de 1917 se regulaba por primera vez el ingreso de los dementes en el pabellón manicomial, donde se admitían pacientes pobres en régimen de observación o reclusión definitiva, y pacientes pensionados "que disfrutarán de otra alimentación y servicio, mediante el pago de una cuota $^{\prime \prime 2}$. Durante el periodo del protectorado español en el norte de Marruecos (1912-1956) el Hospital Civil de Málaga también atendió casos que provenían de esta zona ${ }^{13}$.

Debido al aumento progresivo de pacientes ingresados, el manicomio requirió numerosas reformas en las décadas posteriores a su edificación. Una de estas actuaciones fue habilitar un nuevo espacio para las dementes. En 1909 se construyó la sala Santa Rita o sala $20^{14}$, que correspondía al manicomio de mujeres y se encontraba anexa al edificio principal del Hospital Civil, al nordeste, rompiendo la estructura simétrica de la construcción (Fernández, 2004, p.391). Sin embargo, como veremos más adelante, el pabellón de mujeres necesitó numerosas reformas en las décadas posteriores.

En 1918 se subastó la obra para construir 8 celdas de aislamiento en San Carlos $^{15}$ y en septiembre de 1923 se aprobó un presupuesto para acondicionar dos celdas en el departamento de dementes varones del Hospital Civil con destino a pacientes sujetos a procesos judiciales. Finalmente fueron alojados en las celdas para furiosos, con las modificaciones que propuso la Diputación "con la condición de colocar en cada una de ellas una segunda reja de seguridad y, además, forrar las puertas con chapa de hierro, colocando un cerrojo fuerte con llave en cada celda"16.

Varias fueron las notificaciones que el arquitecto de la corporación realizó durante la década de los años 20, exponiendo el mal estado en el que se encontraba San Carlos. En 1924 la cubierta del pabellón de varones agitados amenazaba ruina por lo que se procedió a aprobar su reparación de forma urgente. Sin embargo, tres años más tarde, en junio de 1927 estas obras seguían sin acometerse, aumentando el riesgo para los pacientes ingresados ${ }^{17}$. En junio de 1926, el arquitecto requirió otras obras en los pabellones de dementes: "Se requiere con urgencia la compostura del pabellón de furiosos, aislamiento del departamento de mujeres dementes construyendo una pequeña consulta, así como la instalación de un departamento de duchas en el de tranquilos y por último reparaciones generales de cubiertas, solerías etc. " ${ }^{18}$. La instalación de una bomba de agua para llevar agua a las salas de dementes fue acometida en $1925^{19}$.

Según el acta de la comisión provincial del 10 de octubre de 1929, se presentó una propuesta para la construcción de un pabellón para dementes sucios varones y otro para niños, contando con el asesoramiento del Dr. Prados Such (Fernández, 2004, p.391), aunque finalizaron las obras cuatro años más tarde, en 1933. Durante los primeros años de la Segunda República se llevaron a cabo obras como la reparación de la red de abastecimiento de aguas y los baños, entre junio y agosto de 1932; también se construyó un nuevo dormitorio en San Carlos, aprovechando el espacio de antiguas celdas de aislamiento, en febrero de 1933; y, entre mayo y octubre del mismo año, se procedió a la instalación de un taller de esparto ${ }^{20}$. Según el reglamento de 1934 del Hospital Civil, existían dos pabellones para hombres: el antiguo (San Carlos) (figuras 2 y 3 ) con una sección para sucios y otra para agitados y epilépticos; y el nuevo pabellón destinado a pacientes tranquilos, con una sección para menores de 10 años $^{21}$. Una consulta externa de psiquiatría o dispensario psiquiátrico comenzó a funcionar en 1931 para el tratamiento ambulatorio de los enfermos ${ }^{22}$, en la línea de los dispensarios creados a partir de los cambios legislativos en la Segunda República Española (Campos y Huertas, 1998, p.108) ${ }^{23}$.

La sala de mujeres precisó ampliación y reformas importantes en 1927, que fueron pagadas con los beneficios obtenidos de una corrida de toros benéfica organizada en la ciudad ${ }^{24}$. Posteriormente, se realizaron nuevas modificaciones desde diciembre de 1931 a agosto de 1932, habilitándose un sótano bajo la sala 14 del hospital general, que fue usado como ampliación de la sala $20^{25}$ (figuras 4 y 5). Pedro Ortiz Ramos, psiquiatra encargado de la sala de mujeres del manicomio, apuntaba: "Antes del 1 de mayo de 1931 existian sólo 90 camas para 130 enfermas. Se ha habilitado un sótano amplísimo capaz para 50 camas y esta instalación se ha hecho en excelentes condiciones () otra mejora ha consistido en sustituir unas arcaicas escaleras de caracol, que conducían al dormitorio del piso alto, por una buena escalera de mármol que da acceso a una amplia galería, antes descubierta y hoy techada, en la que pensamos instalar talleres para la ocupación de las enfermas ( )"26.

Sin embargo, en la memoria que realizó para la diputación en octubre de 1934, Pedro Ortiz Ramos insistía 
Figura 2. ADPM. Lg. 4609, p. 019. Sala San Carlos

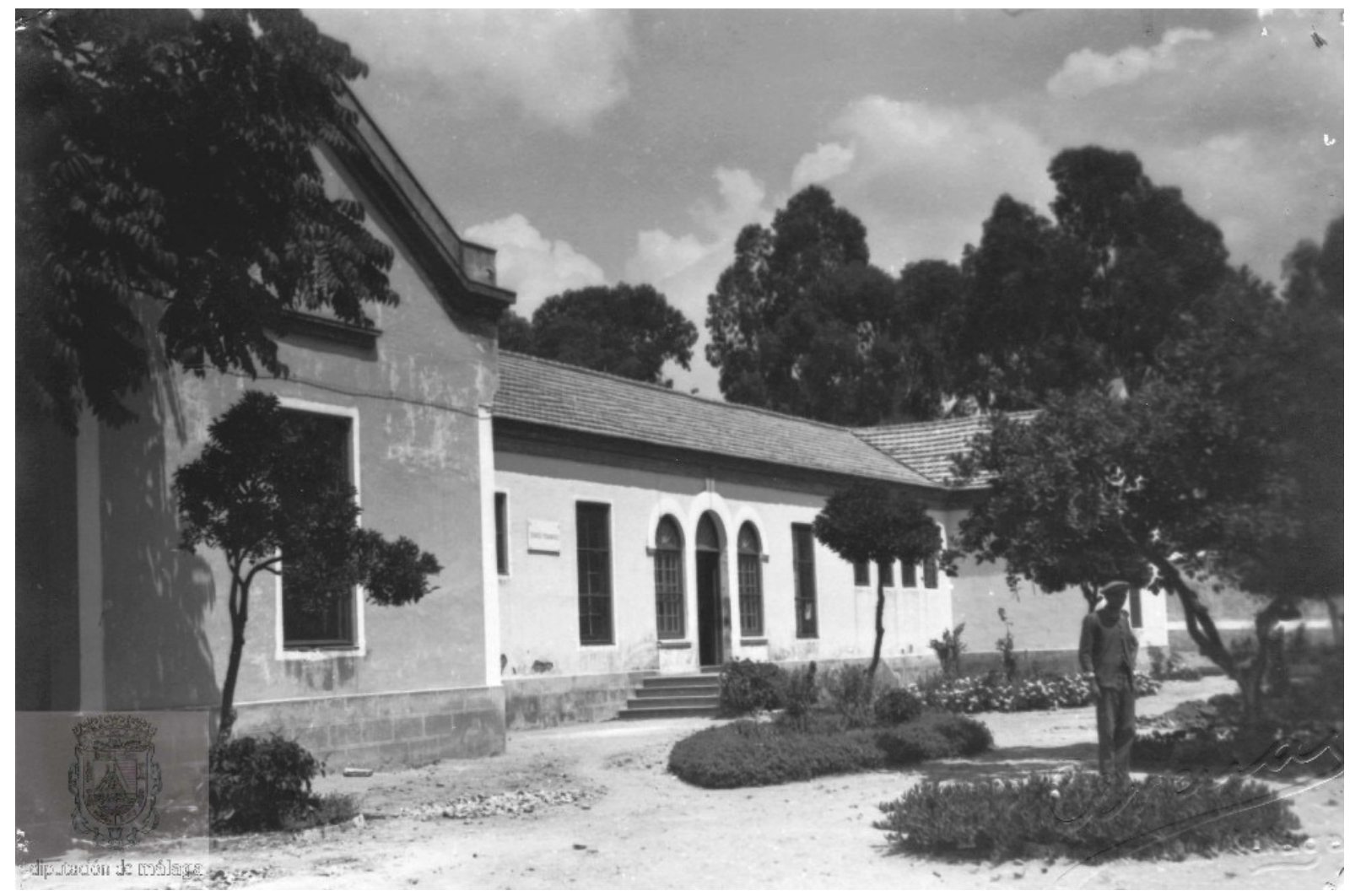

Figura 3. ADPM. Lg. 4609, p. 023. Sala San Carlos

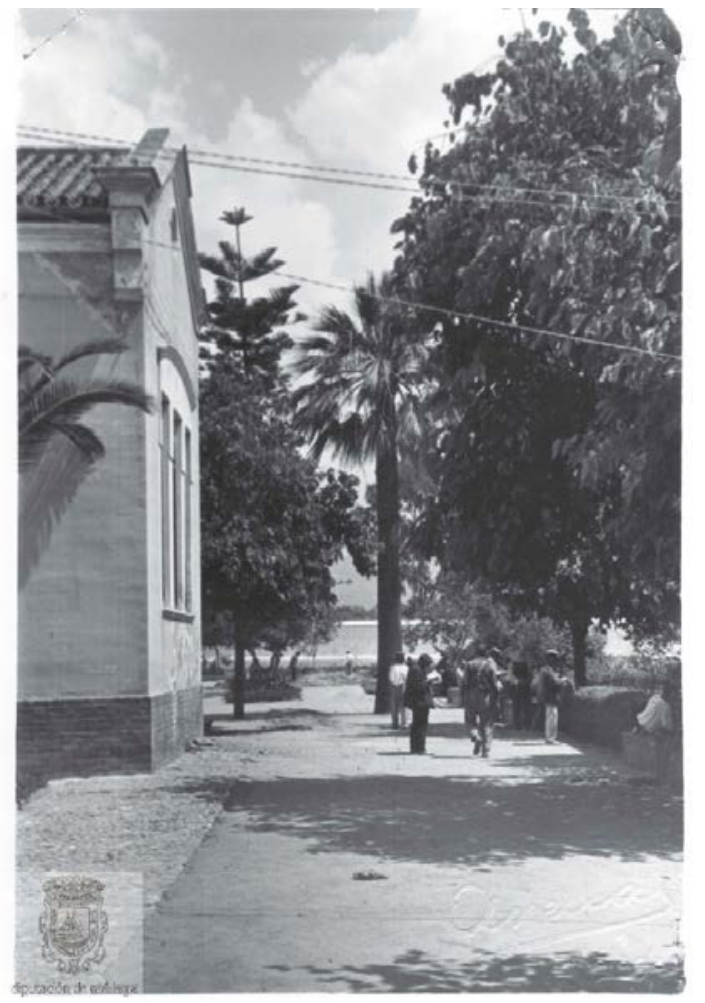


Figura 4. ADPM. Memoria Hospital Civil, 1931-1934, Ig. 4802, p. 36. Sala Santa Rita

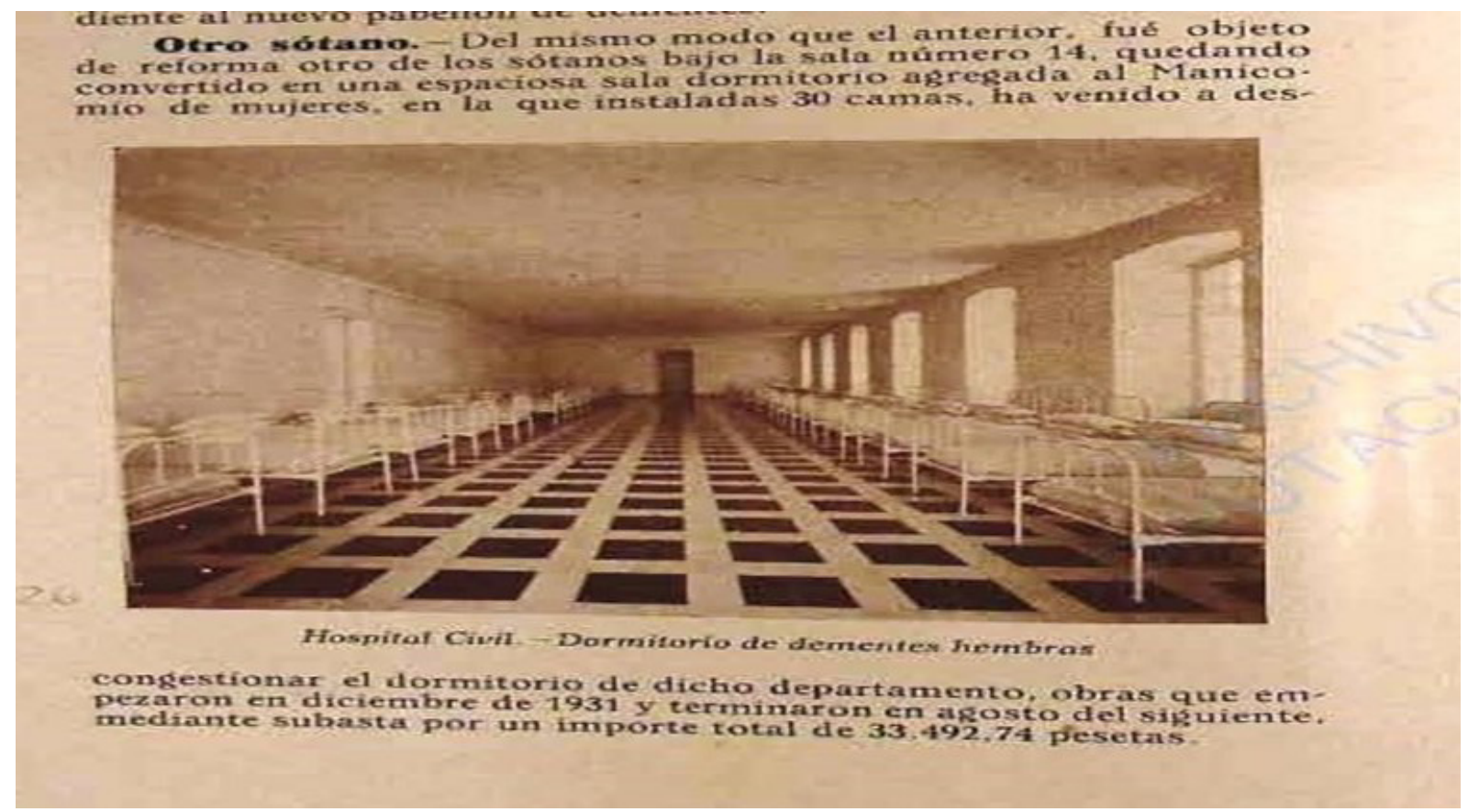

Figura 5. ADPM. Memoria Hospital Civil, 1931-1933, Ig. 4820, p. 39. Sala Santa Rita

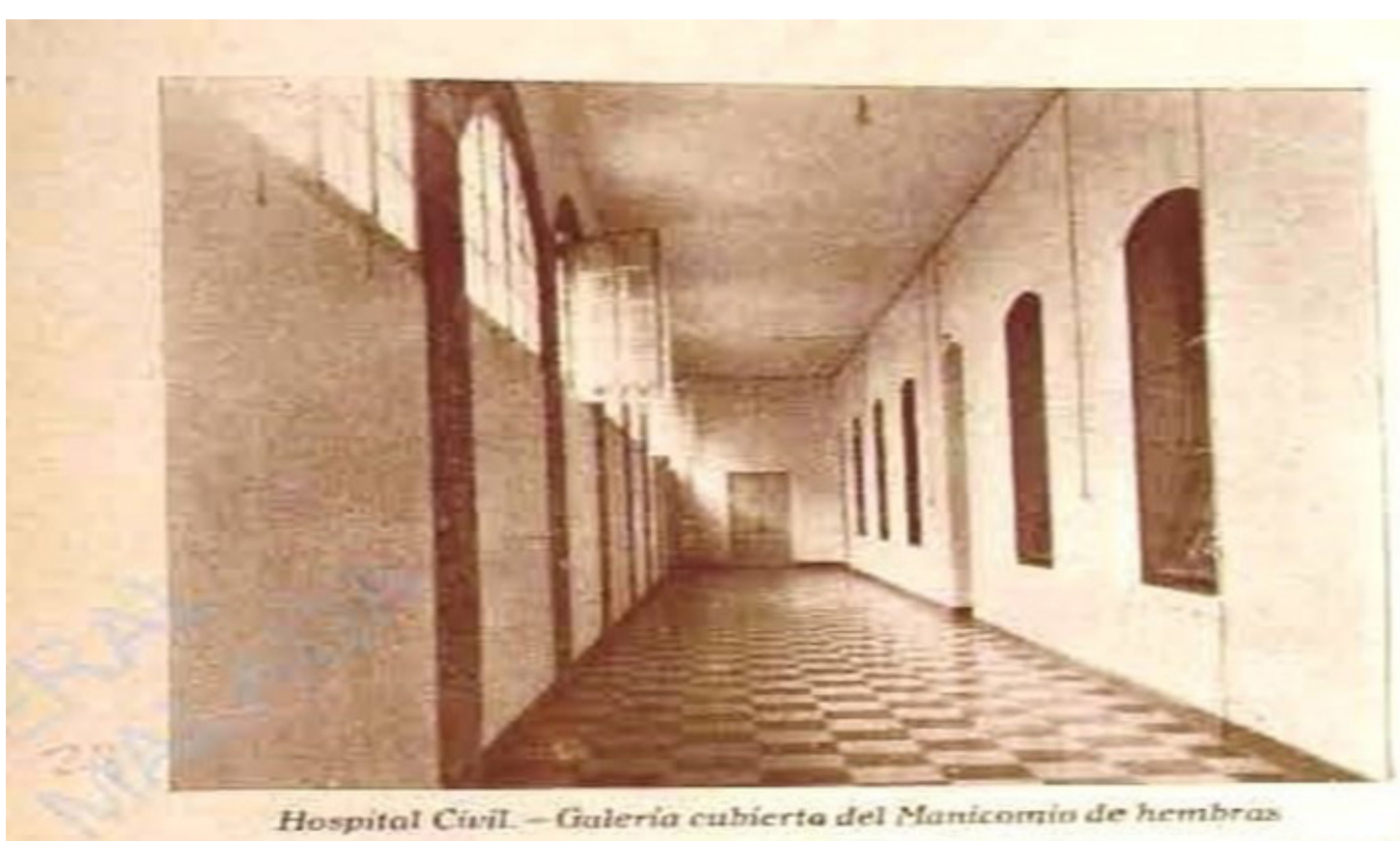

en la sobrecarga de pacientes del pabellón de hombres, que provocaba el hacinamiento de los mismos, y proponía la creación de un pabellón de sucios en el lugar de la leprosería, ya que estaba previsto que los pacientes de lepra fuesen trasladados a la Leprosería
Nacional de Fontilles, con el deseo de que "una vez ello, se habrán convertido en realidad nuestras aspiraciones de que los dementes tengan alojamiento adecuado, cada uno en su cama, en iguales condiciones que los demás hospitalizados en el Establecimiento" 27. 
La situación descrita por Ortiz fue reconocida por la Diputación como el resultado de un aumento progresivo de los ingresos en los últimos años ${ }^{28}$; sin embargo, no quedó reflejada la falta de aceptación por parte de la corporación de ciertos cambios y reformas propuestos por Miguel Prados Such, que tenían la intención de mejorar el manicomio y dar una asistencia más humana a los dementes, cuestión esta que analizaremos más adelante.

\section{LOS PROFESIONALES DE LA NEUROPSIQUIATRÍA EN EL MANICOMIO PROVINCIAL DE MÁLAGA}

La psiquiatría es una especialidad muy relacionada con la dimensión social y cultural, por ello, no se entiende el estudio de esta sin un estudio en profundidad de ambos contextos. Desde esta perspectiva, como apunta Huertas, la conexión entre biografías y aportaciones a la disciplina, se hace necesaria. La familia de origen, los años de formación, destinos laborales, becas, intercambios, conexiones con otros compañeros, la posición ideológica y la sensibilidad social aportan una información valiosa y vienen a explicar el porqué de determinadas propuestas, así como su aceptación o rechazo por parte de la comunidad científica (Huertas, 2002a, p. 15).

Durante la década de los años 20 y 30, hasta la Guerra Civil, se fue conformando en España un proceso de institucionalización y formación de la psiquiatría como especialidad médica (Huertas, 2002b, p.101). En este proceso intervinieron varios acontecimientos: la aparición de la revista Archivos de Neurobiología (1920), el nacimiento de la Asociación Española de Neuropsiquiatras (1924) y de la Liga Española de Higiene Mental (1926), el Decreto sobre asistencia de enfermos mentales (1931), la creación del Consejo Superior Psiquiátrico (1931) y la puesta en marcha de las primeras cátedras de Psiquiatría y Neurología en la Universidad de Barcelona (1933) (Espino, 1997; Lázaro, 1995). Es en este caldo de cultivo profesional en el que se debe incluir los intentos de reforma del Psiquiátrico de Málaga por parte de los ya mencionados Prados Such y Ortiz Ramos. Sin embargo, aunque ambos psiquiatras forman parte de una generación de especialistas en los que pueden identificarse planteamientos y dinámicas similares, un breve perfil biográfico de ambos nos puede ayudar a contextualizar mejor su labor al frente del manicomio malagueño. Tal como sostiene Rafael Huertas, "[ ]la tensión entre lo biográfico y lo social se hace patente cuando intentamos relacionar la vida y la obra de un autor desde una perspectiva que podríamos denominar dialéctica, en la medida en la que intentemos confrontar la experiencia vivida con la producción de conocimiento" (Huertas, 2017a, p. 15). Por tanto, las biografías son relevantes para comprender y explicar las propuestas y actuaciones de quienes han ocupado un lugar en la toma de decisiones, en este caso en el ámbito de la asistencia psiquiátrica.

\subsection{Miguel Prados Such: del laboratorio a la clínica}

Como ya se ha apuntado, Miguel Prados Such jugó un corto pero relevante papel en las reformas del Manicomio Provincial. Nació en Málaga el 8 de octubre de 1894, en el seno de una familia burguesa (hermano del poeta Emilio Prados). Estudió medicina en Madrid obteniendo su graduación en 1920. Fue alumno en la Residencia de Estudiantes de esta ciudad, donde se relacionó con artistas y científicos de la llamada generación del 27. Hablaba y leía inglés, alemán y francés, por lo que pudo acceder de forma temprana a las obras de Freud cuando aún no estaban traducidas al castellano ${ }^{29}$. En 1916, Miguel prestó a su hermano La psychanalyse des neuroses et des psychoses (Régis y Hesnard, 1914) y comenzaron a utilizar el autoanálisis. La influencia psicoanalítica de su hermano es clara en la poesía de Emilio Prados, por ello se le atribuye la difusión de las ideas psicoanalíticas dentro de la generación del 27 durante su estancia en la residencia de estudiantes entre 1910 y 1920 (Lázaro, 2010, p. 27-29). Entre 1918 y 1920 colaboró con Ramón y Cajal y Gonzalo Rodríguez Lafora en el laboratorio de fisiología cerebral que dependía de la Junta de Ampliación de Estudios (JAE) (Linares, 1983, p. 62), participando en las investigaciones sobre líquido cefalorraquídeo que fueron los primeros trabajos en fisiología de Rodríguez Lafora (Rodríguez Lafora y Prados, 1918; Rodríguez Lafora y Prados, 1920). Junto a él también trabajó en el laboratorio de la Residencia de Estudiantes, realizando estudios sobre fisiología y patología del cuerpo calloso, trabajos originales tanto por la temática, como por la metodología que utilizaron. Al mismo tiempo colaboraba en el laboratorio de histopatología de Pío del Rio Hortega (Río-Hortega, 2013, p. 182). En abril de 1920, Miguel Prados obtuvo una beca para viajar a Oxford y Londres con objeto de continuar su formación. Fue en diciembre de 1920 cuando se recibió en la JAE la primera carta de su estancia en Inglaterra. En julio de 1921, según carta remitida desde Londres $^{30}$ se encontraba ya en el Maudsley Hospital de Londres realizando estudios comparativos sobre las glándulas de secreción interna entre los pacientes con demencia precoz y los pacientes con parálisis general progresiva (PGP), bajo la dirección de 
Frederick Mott. No sólo se interesó Prados Such por la investigación, sino que otro motivo de interés para salir de nuestro país lo constituyó el conocimiento de la organización de la asistencia a los alienados, como el mismo Prados reconocía en esta carta: "También he visitado los manicomios públicos del London County Council y pienso redactar una pequeña información sobre su organización que creo pudiera ser de alguna utilidad en España, donde es casi desconocido la asistencia de los alienados en este país"31.

En febrero de 1922 viajó a Múnich, pues, aunque su primera idea era renovar su beca en Inglaterra, finalmente decidió trasladarse a Alemania. Allí colaboró en el laboratorio del profesor Spielmeyer y asistió a las conferencias de Kraepelin, según él mismo relataba en otra carta ${ }^{32}$, ampliando su formación en la histopatología de enfermedades mentales como la psicosis y sobre el líquido cefalorraquídeo con Plaut. También se interesó en histopatología y patogenia del idiotismo infantil amaurótico y fisiología del cuerpo estriado. Un año más tarde, el 8 de marzo de 1923, Miguel Prados escribió una carta desde Múnich a la Junta de Ampliación de Estudios renunciando a los dos meses de beca que le quedaban, para aceptar el puesto de director del Sanatorio Psiquiátrico de San José en Málaga, que fue inaugurado unos días después, el 19 de marzo (Morales, 2012, p. 502). En agosto de 1925, fue nombrado, por la Junta Provincial de la Diputación, profesor supernumerario por oposición del Cuerpo Médico de la Beneficencia. En principio, se encargó de la asistencia de todos los enfermos mentales, tanto hombres como mujeres, pero con la llegada de Pedro Ortiz Ramos, otro psiquiatra clave en la historia del manicomio, segregaron la asistencia psiquiátrica por sexos: Prados Such se adscribió a la sala 21 para hombres y Ortiz Ramos a la sala 20 para mujeres.

Su actividad profesional no se limitó al ámbito local malagueño, sino que siguió vinculado a instituciones nacionales e internacionales. En las Actas de la reunión nacional de neuropsiquiatras de 1924, quedó reflejado que Prados Such se incorporó a la asociación ${ }^{33}$. Además, en los documentos de archivo localizados consta un permiso que Prados Such solicitó en abril de 1926 para ampliar estudios en Italia ${ }^{34}$. Posteriormente, en junio de ese mismo año, acudió a la primera Reunión de la Asociación de Neuropsiquiatras en Barcelona (Lázaro, 2000), participando con una intervención titulada "Plan de organización de los trabajos colectivos que puedan presentarse al Congreso de Washington", de la que eran coautores Rodríguez Lafora y Sanchís Banús ${ }^{35}$. Trabajó y residió en Málaga hasta 1933, año en el que, aunque opositó a la primera cátedra de Psiquiatría de la Universidad Central de Madrid $^{36}$, no consiguió ganarla aun contando con el apoyo de Rodríguez Lafora, quien, décadas más tarde, dedicaba un artículo a la memoria de Miguel Prados Such en los siguientes términos: "Posteriormente, con su individualidad propia, se hizo uno de los mejores conocedores de la Psicopatología de Jaspers, antes de que ésta fuese traducida del alemán al francés, y lució estos conocimientos en sus excelentes oposiciones a la Cátedra de Psiquiatría de Madrid, primera de las que se fundaron en España; oposiciones que, por dispersión de los votos del tribunal entre varios opositores, quedó desierta" (Rodríguez Lafora, 1969, p.473).

Tras ocho años de labor clínica en Málaga, se trasladó a Madrid en 1933, previa petición de una excedencia, para seguir sus investigaciones en el Instituto Ramón y Cajal. Sin duda, sus problemas con la institución malagueña y el personal de asistencia psiquiátrica, que abordaremos a continuación, no fueron ajenos a esta decisión de Prados Such. No volvió Miguel Prados a ejercer como psiquiatra en el Hospital Psiquiátrico Provincial de Málaga ya que, durante la depuración franquista de funcionarios, quedó registrado su cese del cargo, con fecha de 30 de noviembre de 1937, mientras se encontraba en excedencia voluntaria $^{37}$. Con anterioridad, el 23 de noviembre de 1936 había sido evacuado desde Madrid a Valencia junto a un grupo de científicos y creadores como Pío del Río Hortega, José Miguel Sacristán y Antonio Machado (López-Ocón, 2007, p.70). El exilio lo llevó primero a Londres y, finalmente, a Canadá, desde donde inició una extensa y rica correspondencia con su hermano Emilio Prados que nos ha permitido conocer parte de su trayectoria e inquietudes profesionales. En una carta de marzo de 1941, Miguel anunció a su hermano la posibilidad de comenzar a trabajar en la Universidad Mac Gill, tanto en la clínica como en la docencia de la asignatura de psiquiatría ${ }^{38}$; en esta actividad veía no sólo la posibilidad de recoger los frutos de su trabajo, sino también de ayudar a su hermano en la difícil situación económica a la que le había conducido su reciente exilio. Al mes siguiente volvió a escribir a Emilio, ya trabajando en el departamento de neurología y neurocirugía de dicha universidad. Aunque el psicoanálisis había estado presente desde el inicio de su carrera, fue en Canadá donde pudo desarrollarlo, tanto desde la clínica, como desde la investigación. En Montreal fundó el Club de Psicoanálisis, en 1944, que, bajo el liderazgo de William Clifford Scott, pasó a ser la Asociación Psicoanalítica Canadiense (Allodi, 2012, p.8). Se jubiló de su puesto como profesor en la 
Universidad Mc Gill en 1957 (Cleghorn, 1969, p.633) y falleció el 17 de enero de 1969.

\subsection{Pedro Ortiz Ramos: entre la Beneficencia y la asistencia privada}

Como ya he apuntado, Pedro Ortiz Ramos fue el otro introductor de una práctica fundamentada en los conocimientos de la neuropsiquiatría en el Psiquiátrico Provincial de Málaga. Nació en Alhama de Granada el 8 de julio de 1903. Sus padres, al igual que los de Prados, pertenecían a la burguesía local. El 18 de febrero de 1927 fue nombrado profesor de la clase de terceros del cuerpo médico de la Beneficencia Provincial con destino a la atención de dementes del Hospital Provincial de Málaga, del que Miguel Prados Such era entonces director; ambos psiquiatras trabajaron conjuntamente en la institución entre 1928 y 1933. Fue representante de la institución provincial en la reunión que la Liga de Higiene Mental celebró en Granada y en el congreso de Zaragoza de $1930^{39}$. El 10 de agosto de 1931 fue nombrado adscrito a la sala de mujeres del Manicomio Provincial. Conoció a Egas Moniz en la reunión de la Asociación Española de Neuropsiquiatras de $1930^{40}$ celebrada en Zaragoza, lo que le permitió viajar a Lisboa a finales de 1935, concretamente al Hospital de Santa Marta, donde acudió a diversas conferencias y tuvo la oportunidad de conocer a otros psiquiatras interesados en la encefalografía cerebral y la lobotomía (Pérez, 2010).

Durante el periodo republicano de la Guerra Civil en Málaga, Pedro Ortiz, como otros tantos médicos del Hospital Provincial, se trasladó a vivir a las instalaciones del hospital, continuando así su trabajo hasta que, en marzo de 1937, pidió licencia temporal y marchó al frente como Alférez del bando franquista; allí fue herido y tuvo que ser dado de baja por este motivo (Pérez, 2010, p.139). Sin embargo, la dictadura franquista le abrió expediente de depuración, con el resultado de sanción de dos años sin empleo ni sueldo, que fue recurrido por él en marzo de 1938. Ortiz reconoció que perteneció a la Agrupación al Servicio de la República. Pero su indefinición política provocó que, entre las declaraciones recogidas para su expediente, algunos trabajadores del hospital lo asociasen al bando republicano y otros al bando franquista. En el mismo escrito donde él trató de defender su adhesión al régimen franquista, con fecha de marzo de 1937, señalaba: "Varios refugiados derechistas de pueblos estuvieron en calidad de supuestos locos, en el Manicomio Provincial, ellos, a la entrada de nuestro glorioso ejército, me abrazaron con efusión. Sus nombres constan en mi archivo y están a la disposición de usted"41. Finalmente fue restituido en su cargo el 25 de octubre de 1938, aunque suspendido de sueldo por dos años (Zamora, 2009, p.28). En 1941, estuvo pensionado en la Nervem Clinic de Múnich, donde recibió formación sobre el coma insulínico; y en 1945 asistió al Instituto Julio Matos donde, junto al profesor Barahona Fernandes, estudió especialmente los resultados de la leucotomía (Pérez, 2010, p.115) ${ }^{42}$. Sin embargo, Ortiz también manejaba otros supuestos, pues en el Congreso Mundial de Psiquiatría de París de 1950 expuso un trabajo sobre la psicoterapia psicoanalítica (Miguelez, Piñeiro, Louzao, et al, 2016; Jordà, Rey, Angosto, 2007). Las referencias a tratamientos psicoterapéuticos en las historias clínicas de mujeres de la Beneficencia son escasas. Posiblemente, ese tipo de terapéuticas las destinara a sus pacientes privados y tanto la malarioterapia en los años 30 como el coma insulínico y el electroshock fueron los tratamientos de elección para las pacientes pobres ingresadas en la Beneficencia durante las décadas de los años 30 y 40 (García; López, et al, 2013; Villasante, 2003). Ya a mediados de los años 50, ingresó en la Real Academia de Medicina de Granada, y en su discurso titulado "Reflexiones sobre la leucotomía", quedó clara su oposición a la realización de éstas técnicas, por las graves secuelas en la personalidad que ocasionaban a los pacientes (Pérez, 2010, pp.183-205)

Por tanto, fue un hombre con una actividad asistencial e intelectual muy importante: además de trabajar en la Beneficencia, ejerció la medicina privada, primero en su consulta y posteriormente con la creación de la Casa de Reposo los Ángeles en Málaga. A partir de los años 50 también desarrolló su actividad clínica en Gibraltar. En 1965 fue nombrado presidente de la Sociedad Española de Psiquiatría. A los 74 años, el 26 de mayo de 1977, se suicidó en su domicilio de un disparo.

\section{LA REALIDAD Y LA UTOPÍA QUE (NO) PUDO SER: CARENCIAS Y DESARROLLOS DE LA REFORMA PSIQUIÁTRICA EN MÁLAGA}

Con fecha del 27 de junio de 1926, Miguel Prados Such entregó a la Institución de Beneficencia una memoria en la que resumía su intervención en la reunión de la Asociación de Neuropsiquiatras que tuvo lugar ese año en Barcelona. Con posterioridad, el 26 de enero de 1932, fue recibida en la diputación una circular que enviaba el Consejo Superior Psiquiátrico, con directrices para la organización de los manicomios Provinciales, a la que se adjuntaba un ejemplar de la obra de Gonzalo Rodríguez Lafora titulada "Lo que debe ser un Manicomio Provincial" que recogía 
la conferencia del psiquiatra madrileño en la Sociedad de Amigos del País en Málaga y que fue editada en 1931 (Rodríguez Lafora, 1931). Ambos escritos recogen el ideario de los psiquiatras de esta generación, si bien hay que resaltar el esfuerzo que Prados Such tuvo que realizar para que este discurso pudiera calar en la organización y asistencia a los dementes de la institución malagueña.

Miguel Prados mostraba gran preocupación por los alienados, señalando aspectos tan importantes como el concepto de curabilidad de la enfermedad mental, y la sustitución del término "manicomio" por "Hospital Psiquiátrico"43. Estos temas constituyeron una de las estrategias diseñadas por este grupo de psiquiatras para concienciar a la sociedad y a los políticos sobre la importancia del tratamiento de la locura. En este sentido, una de las aportaciones más significativa del escrito de Prados Such fue lo referente a aspectos organizativo-asistenciales, conformando una "utopía asistencial". Utopía en el sentido de ideal asistencial al que tendían, como movimiento que trabajó para reformar el tratamiento y el cuidado de los dementes. La crítica sobre la estricta separación del loco y la sociedad favorecía la visión de los alienados como enfermos que necesitaban asistencia, y desde ahí, se erigía el hospital psiquiátrico como lugar de asistencia y no de reclusión: "El que trata con enfermos recluidos, observa constantemente, sobre todo en los primeros días de la reclusión, como el enfermo, atormentado por sus ideas delirantes pregunta al verse internado en una de las clásicas celdas "¿Qué delito he cometido para que me traigan a ésta cárcel?" y en muchos casos, la observación clínica se haya dificultada ante la creencia del enfermo de encontrarse ante el que le explora, no de un médico, sino de un juez, lo que ve por otra parte confirmado, al recibir por los brutales enfermeros el trato carcelario y desconsiderado de presidio" ${ }^{44}$.

Desde la Restauración monárquica, la idea de la peligrosidad de la enfermedad mental estuvo entrecruzada con el interés de los profesionales de buscar la patologización de conductas, y realizar así tareas higienistas en la sociedad, ya entrado el siglo XX (Campos, 1997; Campos, 2013; Campos, 2016, p. 113). En este sentido, Lafora intentaba justificar la necesidad inmediata de la creación de asilos para su tratamiento, basándose en la peligrosidad, una de las cuestiones que los nuevos psiquiatras usaron para demandar más recursos económicos ya que la prensa sensacionalista de la época se hacía eco de los asesinatos y los actos delictivos a manos de dementes, con especial interés: "España tiene, pues, obligación de atender prontamente esta necesidad, con lo que se evitarían infinitos delitos, suicidios y crímenes brutales, que a diario leemos en los periódicos como realizados por locos" (Rodríguez Lafora, 1931, p. 301).

La lucha de Prados Such por mejorar las condiciones de los dementes comenzó desde su llegada a la institución malagueña. Su investigación sobre la pelagra intramanicomial demostró que parte de los trastornos conductuales sufridos por los pacientes ingresados en la institución de la beneficencia podían relacionarse con la alimentación deficiente que se les proporcionaba (Prados, 1929) ${ }^{45}$. Esto provocó una reacción de desagrado en la Diputación, pero finalmente consiguió que se aumentaran los presupuestos del manicomio para mejorar la alimentación de los pacientes, con la idea de reducir la comorbilidad con la pelagra (Linares, 1983, p.62).

En cuanto a los tratamientos, la hidroterapia constituyó uno de los tratamientos de elección desde que se inició el tratamiento moral hasta la década de los años 30 del siglo XX en España; pero fueron frecuentes las quejas de los profesionales con respecto a los abastecimientos de agua (Conseglieri, 2008, p.144-145), y las instalaciones precarias, circunstancias que Prados Such también señala en su escrito de 1926: "Hasta hace pocos meses y merced a la generosidad privada no ha tenido nuestro manicomio provincial un servicio de baños, que constituye el $A B C$ de todo tratamiento en psiquiatría. Hay que hacer constar que actualmente el servicio de que disponemos es insuficiente, puesto que existen tan solo tres bañeras y el número de enfermos recluidos asciende a más de diez veces de lo que ese número de bañeras puede suministrar. Es necesario, que por lo menos, cada sala tenga un par de baños, a fin de que se puedan utilizar para el aseo de los enfermos además del tratamiento" ${ }^{46}$.

Sin embargo, la introducción de la laborterapia como terapéutica comenzaba a vislumbrarse como el pilar fundamental del tratamiento de los dementes, donde coexistieron, no sólo la visión terapéutica, sino también, una cierta visión economicista. Por un lado, los locos constituían una carga para el sistema social y económico del momento, porque no producían. Por otro lado, desde una visión terapéutica, se insistía en la necesidad de no tenerlos ociosos, sino distraídos y ocupados, con objeto de evitar accesos de agitación durante los que pudieran romper mobiliario y ajuar del manicomio $^{47}$. Lafora mantenía que la laborterapia cambiaría el concepto de las instituciones manicomiales pasando de ser "almacenes de seres recluidos en inactividad y con un constante sentimiento de protesta, a locales 
de trabajo con orden y tranquilidad" (Rodríguez Lafora, 1931, p.307), y describió los tipos de trabajos que podían desempeñar los enfermos en la institución. Los clasificó en tres tipos: domésticos interiores, industriales y agrícolas, señalando que este último, era el más beneficioso para la salud de los enfermos y la economía del establecimiento, ya que sostenía en gran parte, los presupuestos de la institución ${ }^{48}$ (Rodríguez Lafora, 1931, p.306). La idea de que los pacientes pudieran pagar parte de sus gastos realizando trabajos dentro de la institución ya se reflejaba en el reglamento de 1917 del Manicomio Provincial "Los enfermos que no sean pensionistas y cuyo estado físico lo permita, a juicio del director del hospital y durante las horas que éste señale, cultivarán la tierra del parque y labrarán esparto, ayudándose con esa labor al gasto de los vestidos" 49 . Posteriormente, en el reglamento de 1934, bajo el epígrafe "Manufactura de dementes y venta de flores", se señalaba que "el beneficio que se obtenga de todo ello habrá de distribuirse en ropas, tabaco y postres entre los que presten tales servicios ${ }^{\prime \prime 50}$.

Basándose en su experiencia en Inglaterra, Prados Such explicaba en su memoria: "En los hospitales mentales de Londres, todo producto manufacturado de los que los enfermos y la administración utiliza, son fabricados por los mismos enfermos, con manifiesto beneficio de la administración, toda vez que pudiendo competir en calidad con los adquiridos en el comercio libre, apenas si tiene que pagar mano de obra, pues el jornal que paga al enfermo, descontada su pensión, queda reducido a una insignificancia. Su establecimiento en nuestro "manicomio" daría inmediatamente resultados excelentes" ${ }^{11}$.

Si bien en el escrito de Prados Such no se mencionaba a qué se podían dedicar las mujeres, Lafora fue muy explícito, señalando que las actividades para ellas tendrían que ser fabricar lápices, bolsillos, coser, punto marca, los más parecidos a las labores que desempeñaban fuera de la institución, insistiendo en que eran los menos productivos (Rodríguez Lafora, 1931, p.306). Sin embargo, las mujeres trabajaban en la institución en todo lo relacionado con lavandería, planchado, costura o cocina como se evidencia en las historias clínicas analizadas del Psiquiátrico Provincial de Málaga (García y Jiménez, 2010, p.136). La visión de algunas pacientes respecto a la laborterapia ha quedado recogida en varios estudios sobre la subjetividad de las mujeres ingresadas en la institución, destacando fenómenos de resistencia al trabajo no remunerado e incluso señalando la explotación a la que se veían sometidas; otras historias clínicas muestran cómo el que las pacientes trabajaran dentro de la institución se constituyó en sí mismo en síntoma de mejoría (García y Jiménez, 2010; García, 2016). En este sentido, es muy interesante ver cómo existieron dinámicas de exclusión social de mujeres con conductas inapropiadas donde el manicomio emerge como sitio de reclusión / reforma de conductas, como apunta Isabel Jiménez en varios de sus trabajos (Jiménez, 2014; Jiménez y Ruiz, 1999; Jiménez y Ruiz, 1997).

Esta división generizada del trabajo que describió Lafora, condicionó la organización del espacio manicomial: aclaraba que el pabellón de hombres era el que debía estar más cerca de los terrenos agrícolas, relegando a las mujeres a talleres de lavado, cosido y marcado (Rodríguez Lafora, 1931, p.306). Por otro lado, cabría preguntarse, si tan claro era que el trabajo agrícola era considerado como el más indicado para la salud de los enfermos ¿por qué las mujeres accedían menos a los mismos? Para remediar esta situación, Pedro Ortiz Ramos reivindicó en su memoria la creación de un pequeño huerto situado al lado de la sala 20 donde las enfermas pudieran tener un espacio al aire libre ${ }^{52}$.

Por otra parte, el debate en torno a la escasa formación del personal subalterno estuvo presente, desde el inicio, en las reuniones de la generación de los psiquiatras de los Archivos de Neurobiología, señalándose la necesidad de instruirlo con objeto de facilitar que el manicomio dejara de funcionar como un régimen carcelario. Varias son las investigaciones en nuestro país que han aportado valiosa información sobre los intentos de profesionalización del personal subalterno (Siles y García, 1996; Villasante, 2013; Villasante, 2015a; Villasante, 2015b; Duro Sánchez y Villasante, 2016). Rodríguez Lafora criticaba la escasa formación de los enfermeros, que solían ser designados por una junta ajena al desarrollo de esta especialidad clínica, y señalaba lo inútil que resultaba querer mejorar la asistencia sin la formación de este personal. En relación con el cuidado de los enfermos, Lafora también distinguió las labores de uno y otro sexo dentro de la enfermería, restringiendo la actuación de los hombres a las situaciones de peligro con pacientes agitados, y poniendo de manifiesto la preferencia por las enfermeras, "pues su actuación es siempre más delicada y cuidadosa" (Rodríguez Lafora, 1931, p.310).

En este sentido, las ideas reformistas de Prados Such chocaron con un personal dentro de las salas del manicomio muy complejo, compuesto por un lado por monjas que ejercían cuidados morales y se ocupaban 
de valorar la religiosidad de las pacientes; y por otro, personal subalterno, entre los que se encontraban enfermeros ${ }^{53}$, que carecían de la formación necesaria para dar un trato más profesional a los dementes y que estaban habituados a ejercer medidas coercitivas y represivas con los pacientes ${ }^{54}$. El enfermero mayor Fernando Ávila Urbano y el enfermero Fernando Urbano Moreno denunciaron determinados hechos que hacen referencia a una lucha hegemónica entre la psiquiatría decimonónica y la intención reformista de los nuevos neuropsiquiatras. En diciembre de 1932 la Diputación Provincial abrió el expediente titulado "Averiguación de las causas y depuración de las responsabilidades por obras que se han venido haciendo sin permiso de la Corporación en la sección de dementes varones del Hospital Civil Provincial" 55 . Se enviaron varios visitadores de la corporación, para analizar lo ocurrido, y notificaron que las obras habían consistido en la demolición de varios tabiques de las celdas de aislamiento, la eliminación del revestimiento de cuero de las paredes y de varias puertas. Según consta en el expediente, estas obras fueron acometidas por Juan López de Gamarra ${ }^{56}$, un alumno en prácticas que no estaba contratado por la Diputación y que estaba siendo instruido por Prados Such ${ }^{57}$. Se les culpó, tanto al alumno como a Prados Such, de la fuga de varios pacientes durante estas obras. Prados Such respondió a estas acusaciones en una declaración de seis páginas manuscritas. En ella reconocía que, efectivamente, puso al frente de los enfermeros a este alumno, aludiendo a la falta de formación de los antiguos trabajadores y a la falta de personal: "en virtud de esta consideración y pensando única y exclusivamente en la mejora y humanización de la asistencia a los enfermos recluidos en el servicio psiquiátrico de hombres y teniendo en cuenta la falta absoluta de conocimientos y cultura que el actual personal de sirvientes de dicho servicio, así como su número francamente inferior en relación al excesivo número de enfermos, hizo el declarante una distribución de servicios, en los que, con la mayor justicia posible, procuró compaginar los derechos de los enfermos con los derechos de los antiguos sirvientes del servicio y las aspiraciones tanto de los futuros alumnos diplomados como la persona del declarante en mejorar dicha asistencia creyendo hacer al mismo tiempo un beneficio a la Excma. Diputación Provincial al duplicar el número de asistentes sin aumentar el gasto para dicha Corporación" ${ }^{\prime 58}$.

Posteriormente, declaró que realizó tales obras en beneficio de los enfermos, que no tenían espacio alguno para poder dormir y tenían que hacerlo a la intemperie en pleno invierno. Por ello, se acordó verbal- mente iniciar estas reformas, que pretendían crear un espacio donde poder proteger a unos 40 enfermos, al menos, del frío y la lluvia. Cuando se le preguntó sobre la situación de por qué fue eliminado el enguatado de la habitación de agitados, Prados Such argumentó que estaba en muy malas condiciones, que producía un olor nauseabundo, ya que no había sido limpiado nunca. También señalaba que bajo la piel del enguatado los enfermeros actuales escondían instrumentos de tortura, que él mismo había prohibido, pero seguían siendo usados a sus espaldas. Explicaba en la declaración que la lona que sobró del enguatado se reutilizó para hacer colchones a los pacientes, que se quitaron las puertas y los bastidores de unos cuartos de baño para los pacientes que siempre estaban cerrados porque los enfermeros tenían las llaves escondidas y los dedicaban a su uso personal. Todas estas actuaciones, aunque reconoció que las hizo sin solicitar permiso alguno a la corporación, las llevó a cabo con la única intención de mejorar la calidad de la estancia de los pacientes en el hospital. Además, hacía referencia a malos tratos a los pacientes por parte del personal, que no había podido demostrar de forma objetiva por la escasa colaboración de los subalternos ${ }^{59}$.

El día 2 de diciembre de 1932 los "aspirantes a auxiliares de psiquiatría" redactaron una carta al periódico republicano Amanecer donde denunciaban la situación del manicomio ${ }^{60}$. La carta sacaba a la luz los problemas entre los antiguos trabajadores del manicomio y los nuevos, denunciando toda clase de abusos, como el robo de comida a los pacientes, que se usaba para "alimentar a unos cerdos propiedad de un determinado enfermero y que el pan se vendiera a personas extrañas para lucrarse con el dinero" ${ }^{\prime 61}$. También se hacía alusión a la desaparición de elementos como las celdas de castigo "donde morían de sed y hambre seres irresponsables de cualquier hecho que su propia demencia les impulsaban a cometer, y que sucumbían en el silencio después de recibir ignominiosos azotes"62. Los autores, claramente posicionados a favor de la gestión de Prados Such, denunciaban las dificultades que la Diputación interpuso frente a las reformas que el psiquiatra introdujo en el Manicomio: “¿Es posible que al señor Mapelli, presidente de la Excma. Diputación Provincial, no le parezcan bien las nuevas normas establecidas por Don Miguel Prados, reconocido justísimamente como una de las primeras figuras de la psiquiatría en España y que, ateniéndose estrictamente a lo decretado por el Ministerio de la Gobernación recientemente y de acuerdo con el Consejo de Psiquiatría, distribuye convenientemente turnos de alumnos que 
en todo momento presten el consuelo moral y científico a aquellos trescientos sesenta y tantos desgraciados que allí tiene recluidos su fatalidad?"63.

Pedro Ortiz Ramos quedó como Jefe de Servicio tras la partida de Miguel Prados a Madrid en 1933. Siguiendo su misma línea reformista y con la intención de mejorar la asistencia a los pacientes, en el periódico La Unión Mercantil el 17 de abril de 1936 fue publicado un artículo extenso acerca de los cambios que estaban llevando a cabo en el Manicomio Provincial, titulado "De los procedimientos antihumanos y las celdas lóbregas al tratamiento humano y la utilización social de los locos". En este artículo, Pedro Ortiz Ramos se esforzó en dar una imagen de cambio en cuanto a la asistencia de la locura, mostrando cómo había cambiado el espacio y las terapéuticas centradas en la laborterapia, donde describía el ideal del manicomio como fábrica: " iBasta imaginar la maravilla de ver transformado el manicomio en un inmenso taller! En él estaría cada loco con su tema, pero el tema sería el trabajo agrícola, individual o colectivo, y las manufacturas diversas (). Un visitante de una clínica así creería hallarse en una gran fábrica y se preguntaría extrañado "pero ¿dónde están los locos?"64.

\section{CONCLUSIÓN}

Las ideas de los psiquiatras reformistas de los $\mathrm{Ar}$ chivos de Neurobiología fueron asimiladas y puestas en práctica durante la segunda y tercera década en la institución malagueña, aunque no sin ambivalencias, como las mostradas en torno al papel del trabajo de los pacientes dentro de la institución. Miguel Prados Such y Pedro Ortiz Ramos vehiculizaron este cambio ideológico en el Manicomio Provincial de Málaga con la intención de poner en marcha una "utopía" asistencial, donde desarrollar todas aquellas reformas con las que soñaban los psiquiatras de esa generación. Sin embargo, encontraron obstáculos institucionales que dificultaron ese proyecto: una burocracia lenta, donde la organización de la Diputación Provincial hacía que cada decisión debiera pasar por el pleno, marcado por gran retraso en la ejecución de las reformas estructurales; carencias económicas, que hicieron que gran parte de las remodelaciones se sufragaran por donaciones, y la posible falta de voluntad política con respecto al cuidado de los dementes. Además, la existencia de un entramado de subalternos con una forma de trabajo arcaica y sin iniciativa renovadora que se oponía a dichos cambios ante el temor de perder ciertos privilegios, posiblemente influyó en la decisión que tomó Prados Such de iniciar por su cuenta reformas no aprobadas por la corporación, lo que le valió la apertura de un expediente disciplinario. El intento de visibilizar esta situación por parte de los psiquiatras con el apoyo de un grupo de jóvenes aspirantes a auxiliares de psiquiatría, influidos por Prados Such, quedó en el plano de la denuncia, sin que fuera tomada en consideración por parte de la Beneficencia. Tras la marcha de Prados a Madrid, Ortiz Ramos, elogió los cambios que la Diputación había favorecido en la década de los años 30 en el manicomio, aunque siguió insistiendo en todas las reformas que aún faltaban para mejorar la asistencia a los pacientes. Con el inicio de la Guerra Civil, las ilusiones de cambio y futuras remodelaciones en relación con el funcionamiento del Manicomio Provincial quedaron truncadas.

\section{AGRADECIMIENTOS}

Quiero agradecer a Rafael Huertas e Isabel Jiménez la lectura del manuscrito, ya que, con sus aportaciones, lo han mejorado, procurando nuevas vías de reflexión y completando aportes bibliográficos. Además, también quiero agradecer a los/las revisoras de Asclepio que han contribuido al enriquecimiento del artículo con sus sugerencias.

Este artículo ha sido realizado en el marco del proyecto de investigación HAR2014-58699-P financiado por el ministerio de Economía y Competitividad.

\section{NOTAS}

1. Traducido al castellano en 1847.

2. Estos enfermos podían estar en su domicilio, en casa de familiares, o recluidos en hospitales, hospicios u otros dispositivos de reclusión relacionados con órdenes religiosas, entre otros (Álvarez-Uría, 1983, p.120).

3. En esas fechas contaba con un total de 35 pacientes ingresados de ambos sexos.

4. En Sevilla, la primera institución específica dedicada a la locura y organizada bajo la ley de Beneficencia fue el Manicomio de Miraflores, inaugurado en 1890 (Giménez, 2008). En Córdoba, la creación del primer centro específico de tratamiento para dementes fue posterior, a partir de 1927 (Ruiz, 2011). En el caso de Jaén, los dementes eran trasladados a otros hospitales como el de Granada, o San Baudilio de Llobregat, hasta la construcción del Sanatorio Neuropsiquiátrico Los Prados, en 1952 (Hernández, 2009). 
5. El Hospital Civil Provincial (Hospital Civil en adelante) comenzó a construirse en 1864. Las obras estuvieron sujetas a paros prolongados por falta de presupuesto, de lo que se informaba trimestralmente a la Diputación. También se explicitaba la dificultad con el abastecimiento de agua. (Fernández, 2004, p.362-418).

6. Durante el siglo XIX, Málaga fue destino de una burguesía que se estableció en la ciudad con la finalidad de explotar los recursos naturales de la zona (sobre todo, la caña de azúcar) y promover la creación de industrias afines. Gran parte de sus aportaciones económicas sufragaron gastos del manicomio y la leprosería del Hospital Civil. En concreto, las familias Crooke y Larios participaron en memoria de Carlos Larios Martínez, Marqués de Guadiaro, comprando camas y parte del ajuar necesario destinado al manicomio (Fernández, 2004, p. 389)

7. Archivo Municipal de Málaga (AMM en adelante). Caja 900, 465/22; caja 900, 465/24.

8. Este Departamento se habilitó en un convento que había sido transformado en lazareto de observación en 1821, llamado Asilo de los Ángeles.

9. Memoria Diputación Provincial 1877, pg. 17-18. El 6 de junio de 1854 se inauguró el Manicomio de San Baudilio de Llobregat, sobre un convento abandonado que fue habilitado para el tratamiento de dementes a pocos kilómetros de Barcelona. Fue la primera institución psiquiátrica privada importante en España, y con la que mantenían convenios las Diputaciones Provinciales, para el traslado de enfermos psiquiátricos, al carecer éstas de instituciones propias (Rey, 1984). Otras instituciones como la Casa de Dementes de Santa Isabel de Leganés también trasladaron pacientes a este establecimiento en las últimas décadas del siglo XIX (Villasante, 2002).

10. La sala San Antonio del Hospital Civil pertenecía a la especialidad de obstetricia y cirugía ginecológica. En la enfermería asociada a esta sala permanecieron los dementes hasta que se construyó el pabellón manicomial. En 1889 los trabajadores de esta sala pidieron a la corporación que se construyera un muro entre los pacientes psiquiátricos y ellos. Archivo de la Diputación Provincial de Málaga (ADPM en adelante) lg. 208/38.

11. José Delgado Collantes desde el 17 de julio de 1889 hasta el 20 de octubre de 1919 y Francisco Linares Enríquez desde el 22 de septiembre de 1889 hasta el 27 de febrero de 1919. En Libros de personal, ADPM. Ig. 4856, p.238.

12. AMM. Biblioteca Municipal, 22/27. En el último cuarto del s. XIX pacientes pobres y privados coexistían en las mismas instituciones, como en el caso del Manicomio de Leganés, donde se establecían tres clases: pensionistas, mediopensionistas y pobres (Villasante, 2008, p.37).

13. En las historias clínicas del ADPM correspondientes al periodo del protectorado, hay varios casos de mujeres que venían de Larache, y Tetuán, para ser tratadas en el Manicomio Provincial de Málaga (ADPM Ig. 10153-157 y lg. 10468-29).

14. ADPM Ig. 340-1.

15. ADPM Ig. 268-14.

16. ADPM Ig. 674-35.

17. “(...) he observado que la cubierta del pabellón de agitados presenta grandes deformaciones denunciando un estado de ruina de la armadura del referido pabellón, y de no acudir pronto a efectuar las obras de consolidación necesarias podría acarrear perjuicios para los asilados, no solo ya del pabellón indicado, sino que podría extenderse hasta las que se albergan en el patio próximo" ADPM Ig 679-26.

18. ADPM Ig. 679-24.

19. ADMP Ig. 632-4.

20. ADPM Memoria Hospital Civil 1931-1934 lg. 4820 pg. 47.

21. AMM Biblioteca Municipal 22/6.

22. ADPM Memoria Hospital Civil 1931-1934 Ig. 4820, p.53.

23. Este dispensario se creó siguiendo el modelo expuesto en el Decreto de 1931, que dio lugar a la Reforma Psiquiátrica de la Segunda República. El Decreto otorgaba a los jefes facultativos de los establecimientos la potestad de dar el alta o no a los internos en función de su "peligrosidad"; asimismo, si bien el trabajo en el dispensario de higiene mental implicaba el seguimiento poscura de los pacientes dados de alta, dicho concepto de peligrosidad prevaleció en el discurso y las prácticas psiquiátricas. Esta circunstancia permite entender por qué el Decreto de 1931 se mantuvo durante todo el franquismo (Campos, 1997; Huertas, 2016).

24. ADPM Ig.893-62.

25. ADPM Memoria Hospital Civil 1931-1934, Ig. 4820 p. 36

26. ADPM Memoria Hospital Civil 1931-1934 Ig. 4820. p. 50.

27. ADPM. Memoria Hospital Civil, 1931-1934, Ig. 4820 p. 48.

28. ADPM. Memoria Hospital Civil, 1931-1934, Ig. 4820 p.44.

29. La traducción de las Obras completas de Freud al castellano fue realizada por Ballesteros en 1922. Sin embargo, antes de esta publicación, el primer artículo en una revista médica traducido al castellano, pero sin autoría, en torno al psicoanálisis data de febrero-marzo de 1893 en la Gaceta Médica de Granada, y casi, simultáneamente, en la Revista de Ciencias médicas de Barcelona, sólo un mes después de la comunicación preliminar de Breuer y Freud en la revista Neurologisches Centralblätt (Carles y Muñoz 
et al, 2000, p.17-18)

30. En Inglaterra, durante los años 20 y 30, la investigación de Mott y su equipo estuvo centrada en la relación entre las glándulas de secreción interna y la enfermedad mental (Evans y Jones, 2012).

31. Expediente Junta de Ampliación de Estudios (JAE en adelante) Miguel Prados Such, Archivo Residencia de Estudiantes, Madrid. Carta 21 de julio de 1921.

32. Expediente JAE Miguel Prados Such, Archivo Residencia de Estudiantes. Carta febrero de 1922.

33. ADPM, Ig. 718-24. En este sentido, las sociedades han constituido un vehículo de "culturas profesionales" generando discursos concretos, y a su vez, prácticas (Huertas, 2017b, p. 26).

34. ADPM, lg. 796-18.

35. El Congreso Internacional de Higiene Mental se aplazó y tuvieron una nueva oportunidad para presentar el trabajo. Sin embargo, por la falta de acuerdo, en la Reunión de 1927 de la AEN en Madrid, presentaron ponencias separadas. Se publicaron (por separado) en Archivos de Neurobiología en 1928. Finalmente, el famoso congreso se celebró en 1930, pero la delegación española estuvo compuesta por Sacristán, Germain y Rodríguez Arias (ninguno de los que habían hecho las ponencias preparatorias).

36. Si bien la primera cátedra de psiquiatría de la Universidad Central se creó el 7 de julio de 1933, quedó desierta hasta pasada la Guerra Civil. Entre 1933 y 1936 Mira y López se encargó de la primera cátedra de psiquiatría en la Universidad Autónoma de Barcelona. Por tanto, aunque la primera en creación fue la de Madrid, la primera que se ocupó fue la de Barcelona.

37. ADPM, libros de personal, lg. 4857.

38. Archivo Emilio Prados. Fundación Residencia de Estudiantes, Madrid. Carta marzo 1941, 2 b.

39. ADPM, Ig. 774-30

40. Egas Moniz fue nombrado miembro extranjero de la asociación en 1929 y al año siguiente, invitado a la quinta reunión de la asociación. Realizó una conferencia sobre encefalografía cerebral, técnica con la que consiguió en 1949 el Premio Nobel (Lázaro, 2000, p. 427).

41. ADPM. Expediente de depuración franquista Pedro Ortiz Ramos, Ig. 9286:1,9

42. En la biografía escrita por su sobrina destaca otros centros visitados como clínicas psiquiátricas en Freiburg, Guttingan, Colonia y múltiples clínicas universitarias en Suiza, Francia, Italia, EEUU y Japón, si bien no se ha localizado en el archivo documentación que acredite estos permisos.
43. ADPM. Memoria redactada por Miguel Prados Such para la Diputación Provincial de Málaga sobre la reunión de la Asociación Española de Neuropsiquiatras de 1926, lg. 718-24, p. 3.

44. ADPM. Memoria redactada por Miguel Prados Such para la Diputación Provincial de Málaga sobre la reunión de la Asociación Española de Neuropsiquiatras de 1926, lg. 71824, p. 4.

45. El interés sobre la relación entre la enfermedad mental y las carencias nutricionales serían retomados posteriormente en España por Bartolomé Llopis, con elaboraciones sobre la psicosis pelagrosa durante la Guerra Civil (Huertas, 2006).

46. ADPM. Memoria redactada por Miguel Prados Such para la Diputación Provincial de Málaga sobre la reunión de la Asociación Española de Neuropsiquiatras de 1926, Ig. 71824, p.4.

47. ADPM, Ig. 4820, Memoria Hospital Civil, 1931-1934, p.51

48. Si bien es cierto que en la institución malagueña la mayoría de los talleres y ocupaciones estaban destinados al autoabastecimiento, es interesante profundizar en la idea sobre las instituciones psiquiátricas y su transformación en fábricas, con la consecuente "proletarización del paciente psiquiátrico" (Leyton, 2008).

49. AMM. Biblioteca Municipal 22/27.

50. AMM. Biblioteca Municipal 22/6

51. ADPM. Memoria redactada por Miguel Prados Such para la Diputación Provincial de Málaga sobre la reunión de la Asociación Española de Neuropsiquiatras de 1926, Ig. 71824, p. 5.

52. ADPM, Ig. 4820, Memoria Hospital Civil, 1931-1934, p 51.

53. El personal subalterno en el Manicomio Provincial desde 1930 a 1934 estaba clasificado en Enfermeros Mayores (2), enfermeros (11), enfermeras (6), sirvientas veladoras (6) y auxiliares de psiquiatría (6). ADPM lg. 1112:16.

54. “(...) ese personal auxiliar carecía de toda formación sanitaria, siendo en general licenciados del Ejército, por pensarse que la disciplina en éste aprendida era lo único necesario para tratar con los locos" (Linares, 1983, p.59).

55. “Averiguación de las causas y depuración de las responsabilidades por obras que se han venido haciendo sin permiso de la Corporación en la sección de dementes varones del Hospital Civil Provincial". 19 de diciembre de 1932. ADPM, Ig. 774:36, p. 3-4.

56. Fernando Ávila Urbano describió así la situación: "Que los enfermeros obedecen las órdenes de Juan Gamarra por haber recibido indicaciones en tal sentido del Dr. Prados Such, director del departamento. Que este individuo, Juan 
Gamarra, a pesar de no ser enfermero fijo, si no practicante de enfermero colocado allí por Dr. Prados, viene ejerciendo sobre los enfermeros antiguos y fijos una especie de jefatura, a su juicio, injusta". ADPM, Ig. 774:36, p. 3.

57. Según la Orden del ministerio de Gobernación del 11 de mayo de 1932 los establecimientos psiquiátricos podían recibir alumnos aspirantes al diploma de enfermeros psiquiátricos, para adquirir formación teórica y práctica en el tratamiento de los pacientes ingresados. Prados Such también señaló la necesidad de realizar unos exámenes al personal antiguo del manicomio, pero estos se negaron a someterse a dichas pruebas. ADPM, Ig. 774:36, p. 9,10.

58. ADPM., Ig. $774: 36$ p. 10

59. ADPM, Ig. $774: 36$ p. 13.
60. “¿Qué pasa en el manicomio? Las mejoras de trata a los enfermos, ordenadas por la superioridad y puestas en práctica por el Dr. Prados, ¿'van a frustrarse?", Amanecer, 2 diciembre 1932. Este artículo se encontraba en el expediente de depuración de responsabilidades de Prados Such, en: ADPM, Ig. 9220-6.

61. ADPM, lg. 9220-6

62. ADPM, lg. 9220-6

63. ADPM, lg. 9220-6.

64. "De los procedimientos antihumanos y las celdas lóbregas al tratamiento humano y la utilización social de los locos". La Unión Mercantil, 16 de abril de 1936, p. 12. Este artículo se encontraba en el expediente de depuración de responsabilidades de Prados Such, en: ADPM, Ig. 9220-6.

Conseglieri, Ana (2008), "La introducción de nuevas medidas terapéuticas: entre la laborterapia y el electroshock en el manicomio de Santa Isabel", Frenia, 8, pp. 131-160.

Delange, David Alberto (2003), Enfermedad y Sociedad en Málaga. El cólera morbo asiático (1833-1885), Tesis, Universidad de Málaga, España.

Duro Sánchez, Alicia; Villasante, Olga (2016), "La asistencia al enfermo mental" de Luis Valenciano: la profesionalización del cuidado al enfermo mental durante la Segunda República Española", Cultura de cuidados, 20 (44), pp. 51-62.

Espino, Antonio (1997), “La formación del psiquiatra en España". En: Aparicio, Víctor (comp.) Orígenes y fundamentos de la psiquiatría en España, Madrid, ELA, pp. 107-143

Esquirol, Jean-Etienne (1838), Des maladies mentales considérées sous les rapports médical, hygiénique et médico-legal, París, Baillière.

Evans, Bonnie; Jones, Edgar (2012), “Organ extracts and the development of psychiatry: hormonal treatments at the Maudsley Hospital (1923-1938)", Journal of the History of the Behaviour Science, 48 (3), pp. 251-276

Falret, Henri Louis (1852), De la construction et de l'organisation des établissement d'aliénés, París, Bailliére.

Falret, Jean-Pierre (1864), Des maladies mentales et des asiles d'aliénés, París, Baillière et fils.

Fernández, María Dolores (2004), Los hospitales malagueños en los siglos XV-XIX. Historia y arquitectura, Málaga, Servicio de publicaciones de la Diputación Provincial de Málaga.

García, Celia; Jiménez. Isabel (2010), “Género, regulación social y subjetividades. Asimilaciones, complicidades y resistencias 
en torno a la loca (El Manicomio Provincial de Málaga, 19201950)", Frenia, 10, pp. 123-144.

García, Celia; López, Laura; Gutiérrez, Daniel (2013), “Comparativa entre las "mujeres" ingresadas en la beneficencia y las "señoras" del hospital privado Sagrado Corazón. Aproximación a las nuevas terapéuticas a principios de siglo XX en Málaga". En: Simón, David; Gómez, Chus; Cibeira, Alcira; Villasante, Olga (eds.) Razón, locura y sociedad. Una mirada a la historia desde el siglo XXI, Madrid, Asociación Española de Neuropsiquiatría, pp.331-337.

García, Celia (2016) "De la mujer ideal a la loca: psiquiatría, locura y género en las primeras décadas del siglo XX en España". En: Pereira, Ana; Rui, Joao (eds), VI Jonadas Internacionais de Historia da Psiquiatria e Saúde Mental, Coímbra, Universidad de Coímbra, pp. 39-47, [CD-ROM].

Giménez, María del Carmen (2008), "La fundación del manicomio de Miraflores en Sevilla”, Frenia, 8, pp. 161-182.

González Duro, Enrique (1996), Historia de la locura en España, tomo III, Madrid, Temas de Hoy.

Hernández, María (2009), "La creación del manicomio de Jaén: reflexiones históricas para la construcción de un discurso social de la atención psiquiátrica", Norte de salud mental, 33, pp. 87-93.

Huertas, Rafael (2002a), Los médicos de la mente. De la neurología al psicoanálisis, Madrid, Novatores.

Huertas, Rafael (2002b), Organizar y persuadir. Estrategias profesionales y teóricas de legitimación de la medicina mental española (1875-1936), Madrid, Frenia.

Huertas, Rafael (2006), “Hambre, enfermedad y locura: la aportación de Bartolomé Llopis al conocimiento de la psicosis pelagrosa", Frenia, 6, pp. 79-107.

Huertas, Rafael (2008), "Between doctrine and clinical practice: nosography and semiology in the work of Jean-EtienneDominique Esquirol (1772-1840)", History of Psychiatry, 19 (2), pp. 123-140.

Huertas, Rafael (2016) “El modelo de atención psiquiátrica durante el primer franquismo: rupturas y continuidades". En: Campos, Ricardo; González de Pablo, Ángel (eds.) Psiquiatría e higiene mental en el primer franquismo, Madrid, La Catarata, pp. 17-45.

Huertas, Rafael (2017a), “El retorno de los biográfico en la historia de la psiquiatría", En: Matusevich, Daniel (ed.), ¿Quién hace la historia? Biografías de psiquiatras argentinos, Buenos Aires, Letra Viva, pp. 7-20.

Huertas, Rafael (2017b), “En los inicios de la psiquiatría franquista. El congreso Nacional de Neurología y Psiquiatría (Barcelona, 1942)", Dynamis, 37 (1), pp. 23-43.
Jiménez, Isabel; Ruiz, María José (1997), “El discurso de género en los órganos de expresión de la psiquiatría española del cambio de siglo". En: La locura y sus instituciones (actas de la Il jornadas de historia de la psiquiatría), Diputación de Valencia, pp. 267-280.

Jiménez, Isabel; Ruiz, Maria José (1999), "La política de género y la psiquiatría española a principios de S. XX". En: Barral, María José; Magallón, Carmen; Miqueo, Consuelo; Sánchez, Maria Dolores (eds.), Interacciones ciencia género, Discurso y práctica científicas de mujeres, Barcelona, Icaria, pp. 185-206

Jiménez, Isabel (2014), “La exclusión de lo inapropiado e inapropiable. Mujeres y psiquiatría en la España de principios del siglo XX". En: Ballesteros, Rosa; Escudero, Carlota; Postigo, Marta (eds.), Voces consonantes feministas desde las Humanidades, las ciencias sociales y experimentales: Homenaje a la profesora Ana María Montiel Torres, Málaga, Universidad de Málaga, pp. 247-264.

Jordà, Enric; Rey, Antonio; Angosto, Tiburcio (2007), "La psiquiatría franquista y del exilio en el I Congreso Mundial de Psiquiatría. París, 1950". En: Campos, Ricardo; Villasante, Olga; Huertas, Rafael (eds), De la «Edad de Plata» al exilio. Construcción y «Reconstrucción» de la psiquiatría española, Madrid, Frenia, pp. 377-394.

Lázaro, José (1995), “La reunión fundacional de la Asociación Española de Neuropsiquiatras", Revista de la Asociación Española de Neuropsiquiatría, 15 (53), pp. 295-308, [en línea], Disponible en: http://www.revistaaen.es/index.php/aen/article/view/15428/15288 (Consultado el 18/09/2018).

Lázaro, José (2000), "Historia de la Asociación Española de Neuropsiquiatría (1924-1999)", Revista de la Asociación Española de Neuropsiquiatría, 20 (75), pp. 397-515.

Lázaro, Jesús (2010), El secreto creador de Salvador Dalí. El método paranoico-crítico (1927-1937), Madrid, Eutelequia.

Leyton, César (2008), “La ciudad de los locos: industrialización, psiquiatría y cuestión social. Chile 1870-1940”, Frenia, 8, pp. 259-275.

Linares, Antonio (1983), "La psiquiatría andaluza en la primera mitad de nuestro siglo", Archivos de Neurobiología, 46 (1), pp. 59-66.

López-Ocón, Leoncio (2007), "El cultivo de las Ciencias Humanas en el centro de estudios históricos de la JAE", Revista Complutense de Educación, 18 (1), pp. 59-76.

Miguelez, Miguel Ángel; Piñeiro, María; Louzao, María José; Angosto, Tiburcio (2016), "A participaçao dos psiquiatras espanhóis no I congreso mundial de 1950 em París". En: Pereira, Ana; Rui Joao, (eds.), VI Jornadas internacionais de Historia da Psiquiatria e Saúde Mental. Coímbra, Universidad de Coímbra, pp. 83-96, [CD-ROM]. 
Morales, Manuel (2012), "Uso y abuso de los tópicos en historia: La Hacienda de San José (Málaga)", Baética, Estudios de Arte, Geografía e Historia, 34, pp. 491-503.

Navarro, María José (2015), De hospitales para el alma a dispositivos de poder: Arquitectura en las instituciones psiquiátricas aragonesas (1809-1939), Tesis Doctoral, Uned, [en línea], Disponible en: http://e-spacio.uned.es/fez/view/ tesisuned:GeoHis-Mjnavarro (Consultado el 18/09/2018).

Pérez, Maria Jesús (2010) Dr. Pedro Ortiz Ramos. Aproximación histórica a su vida y obra, Málaga, editorial 33.

Peset, Jose Luis (1995), "El manicomio modelo en España". En: Un siglo de psiquiatría en España. Madrid, Extraeditorial.

Prados Such, Miguel (1929) “Contribución al estudio de la pelagra intramanicomial", Archivos de Neurobiología, 9 (2), 168-177.

Régis, Emmanuel; Hesnard, Angelo (1914), La psychoanalyse des neuroses et des psychose, París, Alcán.

Rey, Antonio (1984), "Clásicos de la Psiquiatría Española del Siglo XIX (VI): Antonio Pujadas Mayans (1811-1881)", Revista de la Asociación Española de Neuropsiquiatría, 4 (9), pp. 73-92.

Río-Hortega, Juan (2013), “A propósito de los descubimientos de la microglía y la oligodendroglía: Pio del Río-Hortega y su relación con Achúcarro y Cajal (1914-1934)", Neurosciences and history, 1 (4), pp. 176-190.

Rodríguez Lafora, Gonzalo; Prados, Miguel (1918), "La circulación del líquido cefalorraquídeo", Boletín de la Sociedad Española de Biología 8, pp. 51-55.

Rodríguez Lafora, Gonzalo; Prados, Miguel (1920), “Nuevos métodos de análisis del líquido cefalorraquídeo", Archivos de Neurobiología 1, pp. 80-89.

Rodríguez Lafora, Gonzalo (1931), "Lo que debe ser un manicomio provincial”, Archivos de Neurobiología, 11, pp. 296-315.

Rodríguez Lafora, Gonzalo (1969), "Recuerdos y añoranzas sobre el doctor Miguel Prados Such", Archivos de Neurobiología 32(4), pp. 473-474.

Rodríguez-Méndez, Rafael (1880), "Estadísticas de los manicomios españoles", Gaceta Médica de Cataluña, 3, pp. 651-660.

Ruiz, Carmen (2011), “El hospital psiquiátrico Provincial de Córdoba: estudio de la población manicomial en el pe- riodo (1900-1940)". En: Martínez, Oscar; Sagasti, Nekane; Villasante, Olga (eds.) Del pleistoceno a nuestros días. Contribuciones a la historia de la psiquiatría, Madrid, Asociación Española de Neuropsiquiatría colección Estudios, pp. 221-235.

Siles, José; García, Encarnación (1996), “Origen histórico de la profesionalización de los cuidados mentales: los practicantes, enfermeros y visitadoras psiquiátricas", Enfermería científica, 174 (5), pp. 49-53.

Villasante, Olga (2002), “Las tres primeras décadas de la Casa de Dementes de Santa Isabel de Leganés". Cuadernos Psiquiatría Comunitaria, 2 (2), pp. 139-162

Villasante, Olga (2003), "La malarioterapia en el tratamiento de la parálisis general progresiva: primeras experiencias en España”. En: Fuentenebro, Filiberto; Huertas, Rafael; Valiente, Carmen (eds), Historia de la psiquiatría en Europa. Madrid, Frenia, pp.175-188.

Villasante, Olga (2008), “Orden y norma en el Manicomio de Leganés (1851-1900): el discurrir diario del paciente decimonónico", Frenia, 8, pp. 33-68.

Villasante, Olga (2013), "Primeros intentos de profesionalización de la enfermería psiquiátrica: de la Segunda República a la Posguerra Española". En: Simón, David; Gómez, Chus; Cibeira, Alcira; Villasante, Olga (Eds.), Razón, locura y sociedad. Una mirada a la historia desde el siglo XXI, Madrid, Asociación Española de Neuropsiquiatría, pp 315-329.

Villasante, Olga (2015a),"El manual del enfermero de manicomios (1902) de Vicente Goyanes: preludio de la formación en enfermería psiquiátrica". Revista de la Asociación Española de Neuropsiquiatría, 35 (126), pp. 403-419.

Villasante, Olga (2015b), “La formación de "enfermeros psiquiátricos" durante la posguerra española: a propósito de Jaén (1939-1955)", Norte de salud mental, 13 (53), pp. 93-103.

Zamora, Ana (2009), “La depuración de funcionarios en la Diputación de Málaga durante el periodo franquista de 1937, a la luz de los documentos custodiados en su archivo", Jábega, 99, pp. 15-30, [en línea], Disponible en: http://www. cedma.es/catalogo/jabega.php?num=99 (Consultado el 18/09/2018)

\section{Fuentes de archivo:}

Archivo Diputación Provincial de Málaga (ADPM).

Archivo Municipal de Málaga (AMM).

Archivo Residencia de Estudiantes de Madrid. 